\title{
Was Aufsichtsräte wissen sollten
}

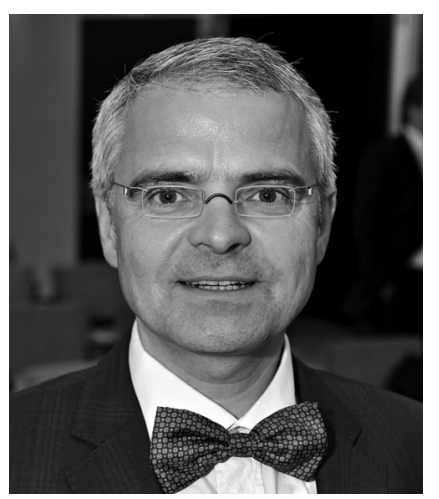

VON FRIEDRICH VOGELBUSCH Prof. Dr. Friedrich Vogelbusch ist Wirtschaftsprüfer und Steuerberater. Er arbeitet als Partner bei der Wirtschaftsprüfungsgesellschaft Warth \& Klein Grant Thornton AG. Er ist Mitglied diverser Aufsichtsgremien - beispielsweise derzeit AufsichtsratsVorsitzender der Bank für Sozialwirtschaft AG - und hat von daher eine eigene Anschauung der Materie. Er ist Honorarprofessor an der Ev. Hochschule für Soziale Arbeit (FH) in Dresden.

www.wkgt.com

\author{
Vier Handbücher wollen das wichtigste Handwerkzeug \\ für Unternehmenskontrolleure in der Wirtschaft \\ zusammenfassen. Inwieweit diese Veröffentlichung \\ auch für Aufsichtsräte in der Sozialwirtschaft geeignet \\ sind, untersucht der nachfolgende Literaturüberblick.
}

In den letzten Jahren ist die Art und Weise, wie Sozialunternehmen ihre Geschäftsführungen beaufsichtigen, wegen aufgedeckter Missstände in der Öffentlichkeit immer wieder diskutiert worden. Auch im Bereich der gewerblichen Wirtschaft und der öffentlichen Unternehmen ist dieses Thema ein Dauerbrenner. Man denke nur an die Diskussion um die Tätigkeit des Aufsichtsrats beim Berliner Flughafen BER!

Zum Aufsichtsrat in der Wirtschaft gibt es vier Buchpublikationen aus den vergangenen Jahren. In diesem Beitrag sollen diese Bücher auf ihre Eignung für in der Sozialwirtschaft tätige Aufsichtsrat besprochen werden.

Der Aufsichtsrat ist vom Gesetz her bei der Rechtsform der Aktiengesellschaft vorgesehen. In der Sozialwirtschaft ist es in den letzten Jahrzehnten üblich geworden, dass auch für die dort häufig genutzten Rechtsformen (e. V., gGmbH, Stiftung) ein Aufsichtsgremium geschaffen wurde. (1)

Nicht erörtert werden in diesem Überblick die Besonderheiten von gemeinnützigen Sozialunternehmen wie etwa die besondere Bedeutung der inhaltlichen Arbeit (Sicherung einer hohen Fachlichkeit und die Vernetzung in die Zivilgesellschaft). (2)

An anderer Stelle hat der Verfasser auf die gestiegenen Anforderungen, die Gesetz und Rechtsprechung an die Aufsichtsrat in gewerblichen Unternehmen stellen, hingewiesen. (3) Neben diesen harten Fakten haben sogenannte Corporate Governance Standards (DCGK) weitere "weiche«Standards gesetzt. In der Wirtschaft kann - bei allen Unzulänglichkeiten, die sich auch in Zukunft mitunter zeigen werden - von einer deutlich professionelleren Aufsichtsrats-Tätigkeit ausgegangen werden.

Nach Ansicht des Verfassers sind auch für Sozialunternehmen Konsequenzen im Hinblick auf eine höhere Professionalisierung der Aufsichtstätigkeit zu ziehen. Persönliche Anforderungen und die Voraussetzung der Unabhängigkeit, wie sie für Aufsichtsrat in der gewerblichen Wirtschaft gelten, sind grundsätzlich auch im Non-Profit-Bereich zu beachten.

\section{"Basiswissen Aufsichtsrat"}

Stephan M. Feil: Basiswissen Aufsichtsrat. Grundlagen einer erfolgreichen Unternehmensüberwachung. Books on Demand 2008. 184 Seiten. 29,95 Euro. ISBN 978-3-8334-9023-1.

Feil legt mit dem Buch »Basiswissen AR « eine wissenschaftlich gehaltene Monografie vor. Es umfasst sieben Kapitel.

Das Buch stellt eine kompakte Übersicht des Themas dar. Neben einem historischen Abriss über die Entstehung und Entwicklung des Aufsichtsrats und des Amts eines Aufsichtsratsmitglieds werden die verschiedenen Ausschussarten und die Akteure des Gremiums - insbesondere Ausschuss- und Gremiumsvorsitzende - näher beschrieben. Feil stellt die jeweiligen Aufgaben, Rechte und Pflichten der Beteiligten, sowie Methoden, Maßstäbe und Tätigkeiten des Überwachungsauftrags dar. Auch die persönlichen und fachlichen Voraussetzungen der Akteure werden erläutert. 
Das Buch eignet sich für den Leser, der sich anhand eines kurzen Kompendiums über das Mindestwissen informieren möchte, das ein Aufsichtsrat in der gewerblichen Wirtschaft bei einer Aktiengesellschaft aufweisen muss. Feil geht nicht auf den Aufsichtsrat im kommunalen oder sozialen Sektor ein.

Vom Layout her ist das Buch wie ein Universitätsskript gehalten (Book on Demand). Der leicht lesbare Text hätte einen namhaften Verlag verdient gehabt.

Das Buch wird keine weitere Auflage erhalten, somit ist die Fortentwicklung des Rechts und der Fachmeinung seit Erscheinen im Jahre 2008 nicht mehr enthalten.

\section{"Der professionelle Aufsichtsrat"}

Peter H. Dehnen (Hg.). Der professionelle Aufsichtsrat. Basiswissen für die Praxis. Ein $360^{\circ}$-Überblick. Frankfurter Allgemeine Buch, Frankfurt am Main 2011. 221 Seiten. 29,90 Euro. ISBN 978-3-89981-255-8.

Das Buch von Dehnen sammelt zwölf Beiträge von verschiedenen Autoren. Der textlichen Darstellung folgt ein Anhang. In diesem Anhang ist der Wortlaut des den Punkt zu bringen, etwa in dem er überspitzt formuliert: »Die Tage der Rotwein-Runden mit alten Freunden und von Kopfnicker-Kreisen sind gezählt.«

\section{"Arbeitshandbuch für Aufsichtsratsmitglieder"}

Johannes Semler, Kersten v. Schenck (Hg.): Arbeitshandbuch für Aufsichtsratsmitglieder. 4. Auflage. C. H. Beck, Verlag Vablen, München 2013. 986 Seiten. 159,- Euro. ISBN 978-3-8006-3442-2.

Das Arbeitshandbuch für AufsichtsratMitglieder ist ein fast tausend Seiten starkes Kompendium zu den für den Aufsichtsrat relevanten Rechtsfragen. Das Buch ist gegliedert in 16 Kapitel. Bemerkenswert ist auch das im Anhang beigefügte Material. Daneben enthält das Buch ein Stichwortverzeichnis (22 Seiten).

Das Arbeitshandbuch stellt eine wertvolle Hilfe für Aufsichtsrat, Vorstände, Richter und Angehörige der beratenden Berufe dar.

Mit diesem Buch kann man sich sicher sein, die wichtigen Gesetzesvorschriften, Gerichtsurteile und Kommentare in der Hand zu halten. Der Verfasser hat zu

\section{"Die Tage von Aufsichtsräten als Rotwein- Runden mit alten Freunden sind gezählt."}

Deutschen Corporate Governance Kodex und Gerichtsurteile abgedruckt, die Aufsichtsrat kennen sollen. Zudem findet sich ein sechsseitiges Glossar.

Der Sammelband beinhaltet einen weit gefassten Überblick über die wichtigsten Funktionen des Aufsichtsrat und wie diese zu erfüllen sind. Dehnen legt als Herausgeber viel Wert darauf, dass die Beiträge mit Beispielen anschaulich argumentieren. Es vermittelt das Basiswissen zu allen relevanten »harten « Themen wie Corporate Governance, Rechte und Pflichten des Aufsichtsrat, Jahresabschlüsse und Insolvenz. Darüber hinaus enthält das Buch auch Beiträge zu den "weichen « Aspekten Wertemanagement, Kommunikation, Rhetorik und der professionelle Außenauftritt.

Rechtsstand des Buches ist das Jahr 2011. Der Sammelband enthält einen dramatischen Appell an die Aufsichtsrat, ihre Arbeit zu professionalisieren. Dehner gelingt es, die Neuerungen auf diversen Fragen das Arbeitshandbuch konsultiert und ist immer auf eine kurze und prägnante Antwort gestoßen.

Für den Aufsichtsrat aus dem sozialen Bereich dürfte dieses Buch ebenfalls unentbehrlich sein.

\section{"Rechte und Pflichten des Aufsichtsrats"}

Marcus Lutter, Gerd Krieger: Rechte und Pflichten des Aufsichtsrats. 5. Auflage. Dr. Otto Schmidt Verlag, Köln 2008561 Seiten. 99,- Euro. ISBN 9783-504-31717-1.

Die Herausgeber dieses Standardkommentars zu Rechten und Pflichten des Aufsichtsrat sind renommierte Gesellschaftsrechtler. Das Werk ist praxisorientiert und gibt einen zusammenhängenden Überblick über die vielfältigen Rechte und Pflichten des Aufsichtsrat und seiner Mitglieder. Dem Buch vorangestellt ist ein ausführliches Inhalts- (27 Seiten) und ein Abkürzungsverzeichnis (6 Seiten) schließt sich an. Das Buch gliedert sich in 20 Kapitel. Am Ende des Buchs ist ein Anhang (10 Seiten), in dem »Die Vergütung des Aufsichtsrats- bzw. Aufsichtsorganmitglieder der DAX 30 Unternehmen « aufgezeigt werden. Daran schließt sich ein Literaturverzeichnis (37 Seiten) sowie ein Stichwortverzeichnis (17 Seiten) an.

Lutter und Krieger haben mit ihrem, mittlerweile in der 5. Auflage erscheinenden Werk, ein praxisorientiertes und bedeutsames Buch verfasst. Besonders gelungen ist der systematische Aufbau des Buches. Hilfreich sind die zahlreichen Hinweise im Text zu Gerichtsurteilen und weiteren Fundstellen. Lutter und Krieger schaffen es, all dies in einer annehmbaren Kürze zu fassen, ohne dabei unwissenschaftlich zu erscheinen, zumal ausführlich auf weiterführende Literatur hingewiesen wird.

Der handbuchähnliche Kommentar ist als Standardlektüre für Aufsichtsrat zu empfehlen.

\section{Fazit}

Die beiden Großkommentare zur Aufsichtsratstätigkeit stellen eine unverzichtbare Arbeitsgrundlage für die professionelle Tätigkeit in einem Aufsichtsrat dar. Zumindest der Aufsichtsratsvorsitzende sollte auf eines der beiden Werke zurückgreifen können. Die Dissertation von Feil ist eine kurze und prägnante Wiedergabe der Materie, gut geeignete für einen ersten Einstieg. Der Sammelband von Dehnen enthält eine breit belichtete Momentaufnahme der verschärften Anforderungen an den Aufsichtsrat, welche die gestiegenen Anforderungen deutlich macht.

\section{Anmerkungen}

(1) Zur Bezeichnung ist anzumerken, dass beim Verein das Aufsichtsgremium oft als »Vorstand «, bei der gGmbH als »Beirat«, bei der Stiftung »Kuratorium « oder »Verwaltungsrat « bezeichnet wird.

(2) Für eine solche Betrachtung vgl. Vogelbusch (2013), Verschärfte Anforderungen an Mitglieder von Aufsichtsgremien in Non Profit Unternehmen, npor 3/2013, S. $132 \mathrm{f}$.

(3) Vgl. Vogelbusch (2013) a. a. O, S. $130 \mathrm{ff}$. 